suggest that BAL taken on day 0 of ARDS upregulated IL17 expression in normal $\mathrm{T}$ cells. This finding was blocked by exogenous 1,25-OH Vitamin D. By contrast, BAL taken on day 4 upregulated FoxP3 and CD25 expression, suggesting an increase in regulatory $\mathrm{T}$ cell activity.

Discussion These results suggest that in early ARDS an imbalance in T cells favouring expression of IL-17 may play a role in the inflammatory response to injury, and this may be attenuated by adequate vitamin $\mathrm{D}$ levels. Later in the course of the disease, Treg cells may predominate and play a role in resolution.

\section{S50 EVALUATION OF SECRETORY LEUCOPROTEASE INHIBITOR (SLPI) AS AN ANTI-INFLAMMATORY THERAPY FOR DONOR LUNG INFLAMMATION}

doi:10.1136/thx.2010.150938.1

${ }^{1} \mathrm{H}$ R Walden, ${ }^{1} \mathrm{D}$ M Karamanou, ${ }^{1} \mathrm{C}$ R Fox, ${ }^{1} \mathrm{~A}$ J Rostron, ${ }^{1} \mathrm{~J}$ A Kirby, ${ }^{2} \mathrm{~A} J$ Simpson, ${ }^{1} \mathrm{~J}$ H Dark, ${ }^{1} \mathrm{~A} J$ Fisher. ${ }^{1}$ University of Newcastle, Newcastle-upon-Tyne, UK; ${ }^{2}$ University of Edinburgh, Edinburgh, UK

Introduction Donor lung inflammation, reflected by high concentrations of interleukin-8 in bronchoalveolar lavage (BAL) and an imbalance between pro-inflammatory IL- 6 and anti inflammatory IL-10 in tissue, correlates with poor graft function and reduced survival after human lung transplantation. Secretory leucoprotease inhibitor (SLPI) is an anti-protease abundant in the lung. SLPI inhibits neutrophil elastase and down regulates inflammatory cytokine transcription via the NFKB pathway. We investigated the effect of SLPI on inflammatory mediators, in a rat model of brain death induced lung injury.

Methods Brain death (BD) was induced in anesthetised ventilated male Wistar rats $(n=16)$ by rapid inflation of an intracranial balloon, the balloon was not inflated in non-BD sham animals. Rats received intra-tracheal human recombinant SLPI $(400 \mathrm{ng} / \mathrm{g})(\mathrm{n}=8)$ or saline (control $\mathrm{n}=8$, sham $\mathrm{n}=9$ ) at $1 \mathrm{~h}$. The experiment was terminated at $5 \mathrm{~h}$. Serum samples were taken at $0,1,3$ and $5 \mathrm{~h}$, and BAL from one lung was taken for cytokine analysis. The second lung was used for wet/dry ratio and Q-PCR analysis.

Results CXCL1, TNF- $\alpha$, IFN- $\gamma$ and IL- 6 were significantly higher in $\mathrm{BAL}$ and serum of control than sham rats, demonstrating that $\mathrm{BD}$ induced lung inflammation in this model. There was no change in lung wet-dry ratio between SLPI treated and control groups. Surprisingly, CXCL1 levels were higher in the BAL of SLPI treated rats than controls $(p=0.05$ ), however no significant difference was

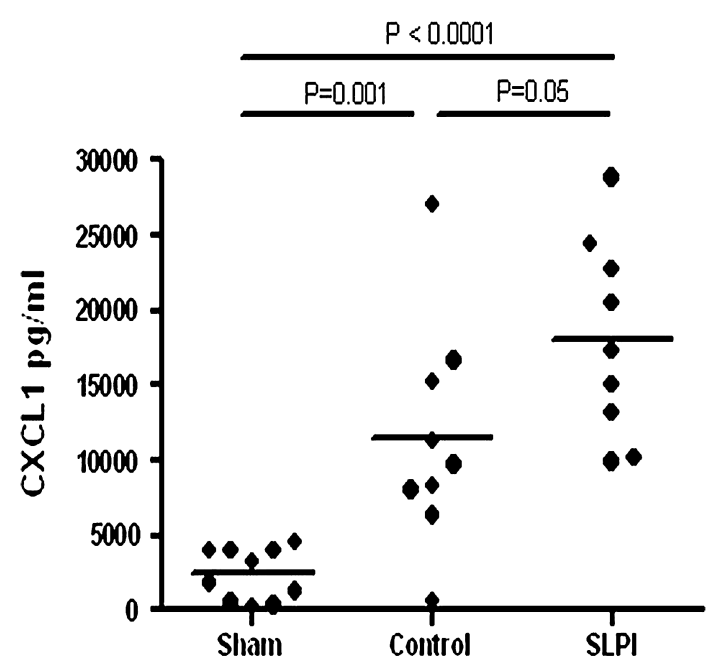

Abstract S50 Figure 1 detected for any other cytokine. There was a non-significant trend towards a higher number of CD45+ leucocytes in BAL in SLPI treated rats compared to controls. CXCL1 mRNA was also increased 1.5 -fold in the SLPI treated group compared to controls $(p<0.05)$.

Conclusions In this study, SLPI does not appear to have an antiinflammatory effect in the rat lung, and may exacerbate inflammation as seen by an increased concentration of the chemokine CXCL1. This surprising effect may be due to the short time course of this experiment where the initial effect of SLPI may be proinflammatory. Given the small window of opportunity available to treat donor lungs we believe that SLPI is an inappropriate intervention for use in lung transplantation.

\section{S51 THIOREDOXIN MODIFIES MIF RELEASE FROM HUMAN MONOCYTES FOLLOWING STIMULATION WITH LTA AND LPS}

doi:10.1136/thx.2010.150938.2

${ }^{1} \mathrm{~S}$ K Leaver, ${ }^{1} \mathrm{G}$ J Quinlan, ${ }^{2} \mathrm{~T}$ W Evans, ${ }^{1} \mathrm{~A}$ Burke-Gaffney. ${ }^{1}$ Unit of Critical Care, National Heart and Lung Institution, Imperial College, London, UK; ${ }^{2}$ Adult Intensive Care Unit, NIHR Respiratory Disease Biomedical Research Unit, Royal Brompton and Harefield NHS Foundation Trust, London, UK

Rationale Thioredoxin ( $\operatorname{Trx})$ is a $12-\mathrm{kDa}$ ubiquitous redox-active thiol (-SH) protein. Plasma levels of Trx are raised in numerous medical and surgical conditions associated with oxidative stress and inflammation such as sepsis (Burke-Gaffney 2005). Trx is thought to have an anti-inflammatory role, at least when released into the circulation. By contrast macrophage migration inhibitory factor (MIF) is regarded as a pivotal pro-inflammatory protein. Indeed co-injection of MIF and $E$. coli enhanced lethality, whereas, anti-MIF monoclonal antibodies conferred protection against murine caecal ligation and puncture and administration of $E$. coli (Calandra 2000). We have previously reported a positive correlation

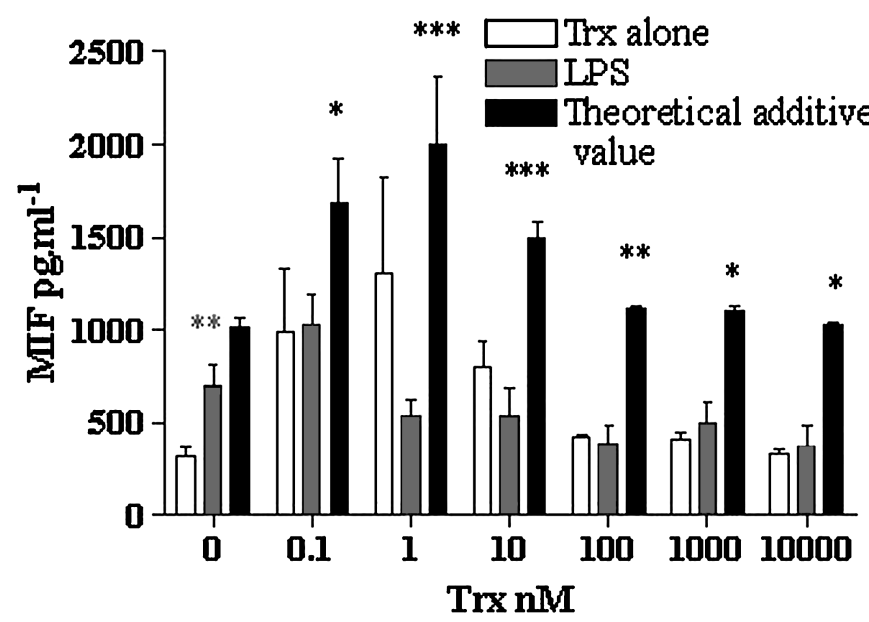

Abstract S51 Figure 1 Effect of Trx of on release of MIF from human monocytes following stimulation with LPS. Human monocytes $\left(1 \times 10^{6} \mathrm{ml}^{-1}\right)$ we re pre-incubated with $\operatorname{Trx}(0.1-10000 \mathrm{mM})$ for $24 \mathrm{~h}$ followed by stimulation with medium alone (white bars) or LPS $1 \mu \mathrm{g} / \mathrm{ml}$ (red bars) for $24 \mathrm{~h}$ MIF concentrations in cell supematants were determined by sandwich ELISA. The black bars represent the theoretical additive values of Trx and LPS. Data represent mean \pm SEM from 5 experiments (LPS) and 3 experiments nts (no treatment). ${ }^{*} p<0.05,{ }^{* *} p$ $\left(0.01,{ }^{* *} p<0.001\right.$ when conditions compared (two way ANOVA with Bonferroni's post test). Red asterisks represent significant increase following LPS alone. Black asterisks represent significant difference between LPS and Trx with the theoretical additive value. MIF, macropage migration inhubitory factor; LPS, lipopolysac charide; LTA, lipoteichoic acid; Trx, Thioredoxin. 